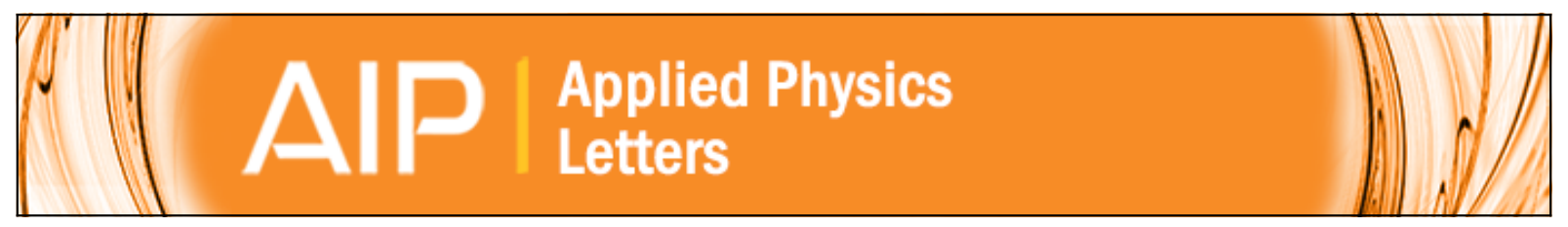

\title{
Rhodium-oxide-coated indium tin oxide for enhancement of hole injection in organic light emitting diodes
}

Soo Young Kim, Jeong Min Baik, Hak Ki Yu, Kwang Young Kim, Yoon-Heung Tak, and Jong-Lam Lee

Citation: Applied Physics Letters 87, 072105 (2005); doi: 10.1063/1.2012534

View online: http://dx.doi.org/10.1063/1.2012534

View Table of Contents: http://scitation.aip.org/content/aip/journal/apl/87/7?ver=pdfcov

Published by the AIP Publishing

\section{Articles you may be interested in}

Efficient and reliable green organic light-emitting diodes with $\mathrm{Cl} 2$ plasma-etched indium tin oxide anode J. Appl. Phys. 112, 013103 (2012); 10.1063/1.4731713

Stabilization of the work function of indium tin oxide using organic surface modifiers in organic light-emitting diodes

Appl. Phys. Lett. 93, 163308 (2008); 10.1063/1.2998599

Enhancement of hole injection using ozone treated Ag nanodots dispersed on indium tin oxide anode for organic light emitting diodes

Appl. Phys. Lett. 90, 163516 (2007); 10.1063/1.2719153

Enhancement of hole injection using iridium-oxide-coated indium tin oxide anodes in organic light-emitting diodes Appl. Phys. Lett. 86, 133504 (2005); 10.1063/1.1894605

Enhancement of hole injection using $\mathrm{O} 2$ plasma-treated $\mathrm{Ag}$ anode for top-emitting organic light-emitting diodes Appl. Phys. Lett. 86, 012104 (2005); 10.1063/1.1846149

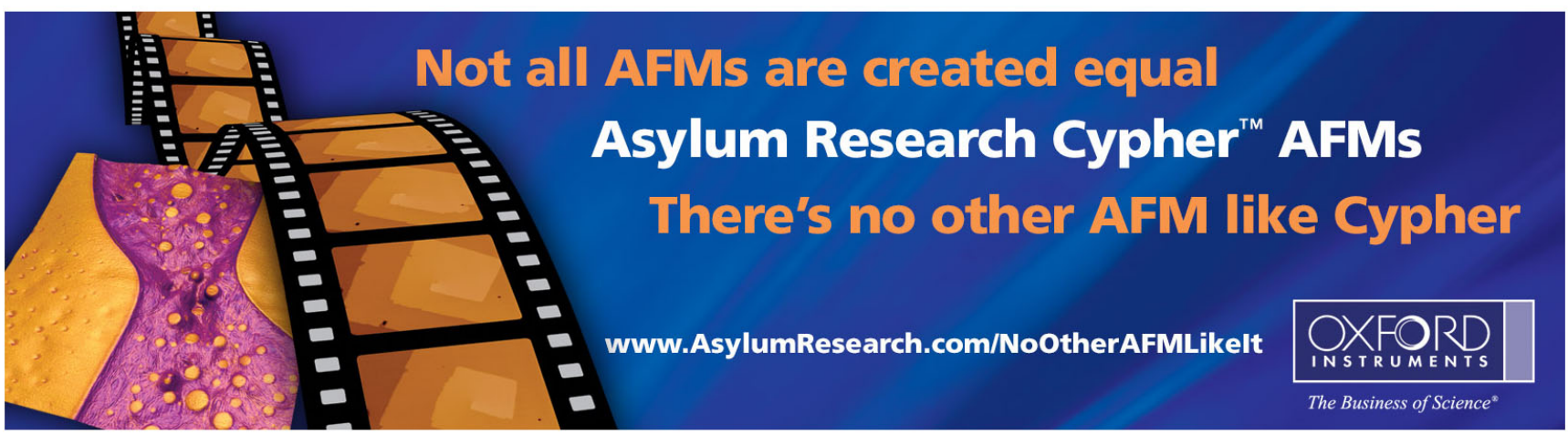




\title{
Rhodium-oxide-coated indium tin oxide for enhancement of hole injection in organic light emitting diodes
}

\author{
Soo Young Kim, Jeong Min Baik, and Hak Ki Yu \\ Department of Materials Science and Engineering, Pohang University of Science and Technology \\ (POSTECH), Pohang, Kyungbuk 790-784, Korea \\ Kwang Young Kim and Yoon-Heung Tak \\ LG Electronics Inc., Kumi, Kyungbuk, 730-030, Korea \\ Jong-Lam Lee ${ }^{\text {a) }}$ \\ Department of Materials Science and Engineering, Pohang University of Science and Technology \\ (POSTECH), Pohang, Kyungbuk 790-784, Korea
}

(Received 15 March 2005; accepted 13 July 2005; published online 12 August 2005)

\begin{abstract}
The authors report the enhancement of hole injection using an $\mathrm{RhO}_{x}$ layer between indium tin oxide anodes and 4,4'-bis[N-(1-naphtyl)- $N$-phenyl-amino]biphenyl in organic light-emitting diodes (OLEDs). The operation voltage of OLEDs at $700 \mathrm{~cd} / \mathrm{m}^{2}$ decreased from 13 to $10 \mathrm{~V}$ as the $\mathrm{Rh}$ layer changed to $\mathrm{RhO}_{x}$ by surface treatment using $\mathrm{O}_{2}$ plasma. Synchrotron radiation photoelectron spectroscopy results showed that the work function increased by $0.2 \mathrm{eV}$ as the Rh layer transformed into $\mathrm{RhO}_{x}$. Thus, the hole injection energy barrier was lowered, reducing the turn-on voltage and increasing the quantum efficiency of OLEDs. () 2005 American Institute of Physics.
\end{abstract}

[DOI: $10.1063 / 1.2012534]$

Ever since the high efficiency organic light-emitting diodes (OLEDs) were reported, numerous efforts have been made to improve OLED characteristics. The performance of an OLED is influenced by the properties of the injecting electrodes and the electrode-organic interfaces. ${ }^{1}$ The potential barrier between electrodes and organic layers exists due to the energy level difference. The barrier for electron injection subsequently reduced using electron injection promoters, such as lithium and cesium fluorides. ${ }^{2,3}$ At the interface of an indium tin oxide (ITO) anode with organic materials, enhanced hole injection was also desired to increase internal quantum efficiency by balancing charge carriers in the active layer. One of the most well-known approaches for reducing potential barriers for hole injection is the elevation of work function of ITO anodes. ${ }^{4-6}$ Several approaches for achieving an elevated ITO work function have been performed to modify its surface chemical states, including surface plasma treatment, the insertion of metal oxides with a high work function between ITO and organic material, and the formation of a metal-doped indium tin oxide layer on an ITO surface. ${ }^{6-8}$ Rhodium oxide $\left(\mathrm{RhO}_{x}\right)$ is a transparent conducting oxide. The work function of $\operatorname{RhO}_{x}(\sim 5.0 \mathrm{eV})$ is higher than that of ITO $(\sim 4.7 \mathrm{eV}){ }^{9}$ Thus, it is expected that the interfacial layer of $\mathrm{RhO}_{x}$ between ITO anodes and organic materials could improve the injection of holes via enhanced OLED electrical properties.

In this letter, we report the enhancement of electrical and optical performance of OLEDs by using an interfacial layer of $\mathrm{RhO}_{x}$ between ITO anodes and the hole transport layer of 4'-bis[N-(1-naphtyl)- $N$-phenyl-amino]biphenyl $(\alpha$-NPD). The $\mathrm{RhO}_{x}$ layer was prepared by exposing a thin $\mathrm{Rh}$ layer to $\mathrm{O}_{2}$ plasma. The $\mathrm{x}$-ray reflectivity method was used to examine the thickness of the thin Rh layer and relative roughness. The change in work function with the formation of $\mathrm{RhO}_{x}$ was

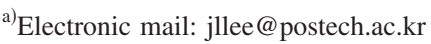

examined using synchrotron radiation photoelectron spectroscopy (SRPES). From this, the effects of $\mathrm{RhO}_{x}$ layers on the enhancement of both electrical and optical properties of OLEDs were discussed.

A glass coating with ITO $(150 \mathrm{~nm}$ thick, $\sim 20 \Omega / \square)$ was used as the starting substrate. The ITO surface was cleaned in sequence with acetone, isopropyl alcohol and deionized water, and then dried with a high purity nitrogen gas. The ITO surface was treated with $\mathrm{O}_{2}$ plasma for 1 min under 100 mTorr ("ITO"). The plasma power was $150 \mathrm{~W}$. A thin layer of Rh with a thickness of $2 \mathrm{~nm}$ was deposited on the plasmatreated ITO by an $e$-beam evaporator ("Rh/ITO"). The Rh film was also exposed to the $\mathrm{O}_{2}$ plasma for $1 \mathrm{~min}$ to produce a $\mathrm{RhO}_{x}$ layer ("O ${ }_{2}-\mathrm{Rh} / \mathrm{ITO}$ "). The thickness of the thin layer was deduced from the period of the oscillations in X-ray reflection, measured at the $3 \mathrm{C} 2$ beamline of the Pohang Acceleration Laboratory (PAL). These three types of samples were loaded into a thermal evaporator and $\alpha$-NPD with a thickness of $70 \mathrm{~nm}$, tris (8-hydroxyquinoline) aluminum $\left(\mathrm{Alq}_{3}, 60 \mathrm{~nm}\right)$, and aluminum $(\mathrm{Al}, 150 \mathrm{~nm}$ ) layers were deposited in sequence. During deposition, the base pressure of the chamber was maintained as low as $10^{-6}$ Torr. The active area of the device was $3 \times 3 \mathrm{~mm}^{2}$. The current density-voltage and luminescence-voltage characteristics of the devices were measured. The surface treatment conditions for the three kinds of anodes are summarized in Table I.

$\mathrm{X}$-ray reflectivity was used to measure the exact thickness of the Rh layer and Rh surface morphology. In order to

TABLE I. Anode preparation methods for OLEDs.

\begin{tabular}{cc}
\hline \hline & OLED anodes \\
\hline ITO & ITO $+\mathrm{O}_{2}$ plasma treatment \\
$\mathrm{Rh} / \mathrm{ITO}$ & $\mathrm{ITO}+\mathrm{O}_{2}$ plasma treatment $+\mathrm{Rh}$ deposition \\
$\mathrm{O}_{2}-\mathrm{Rh} / \mathrm{ITO}$ & $\mathrm{ITO}+\mathrm{O}_{2}$ plasma treatment $+\mathrm{Rh}$ deposition $+\mathrm{O}_{2}$ \\
& plasma treatment \\
\hline \hline
\end{tabular}



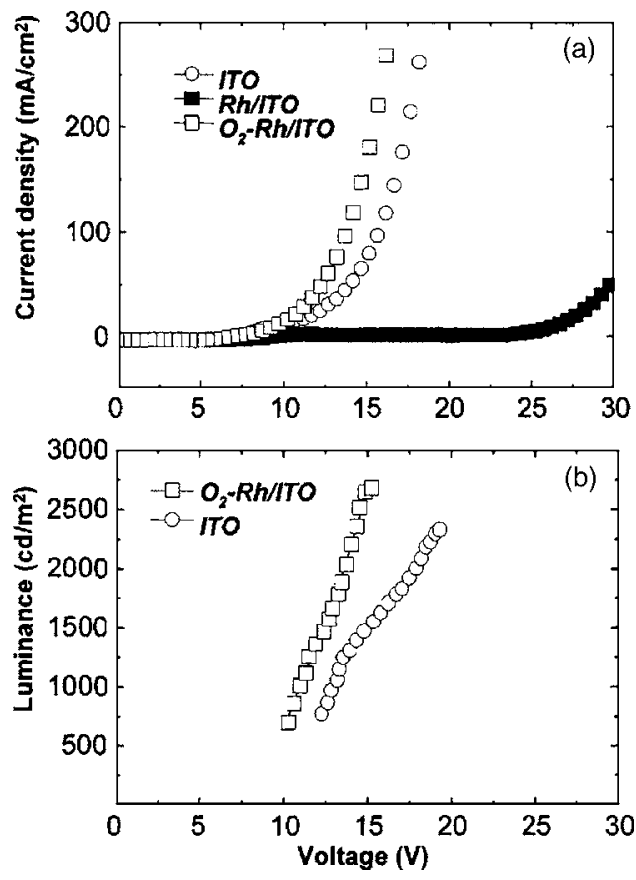

FIG. 1. (a) Current density-voltage and (b) luminance-voltage characteristics of OLEDs with different anodes.

investigate the chemical bonding states of ITO by inserting the $\mathrm{RhO}_{x}$ interfacial layer, the three samples were loaded into a vacuum chamber, equipped with an electron analyzer, at the PAL 4B1 beamline. An incident photon energy of $650 \mathrm{eV}$ was used to obtain Rh $3 d$, In $3 d$, Sn $3 d$, O $1 s$, and $\mathrm{C} 1 s$ core level spectra. The onset of photoemission, corresponding to the vacuum level at the ITO surface, was measured with a negative bias $(-20 \mathrm{~V})$ on the sample to avoid the work function of the detector. The incident photon energy was calibrated with the core level spectrum of $\mathrm{Au} 4 f$.

Figure 1(a) shows the current density-voltage characteristics of the three types of devices. The turn-on voltage increased from 7 to $25 \mathrm{~V}$ when the $2 \mathrm{~nm}$ thick Rh was deposited on ITO. However, it was drastically decreased to $5 \mathrm{~V}$ as the $\mathrm{Rh}$ layer was treated with $\mathrm{O}_{2}$ plasma. The decrease in turn-on voltage is a reflection of improved hole injection efficiency. Luminance-voltage curves are shown in Fig. 1(b). The operation voltage corresponding to $700 \mathrm{~cd} / \mathrm{m}^{2}$ was $13 \mathrm{~V}$ for ITO and $10 \mathrm{~V}$ for $\mathrm{O}_{2}-\mathrm{Rh} / \mathrm{ITO}$. The external quantum efficiencies at $700 \mathrm{~cd} / \mathrm{m}^{2}$ are calculated at $1.1 \%$ for $\mathrm{O}_{2}$ - Rh/ITO and $0.34 \%$ for ITO, respectively. The electroluminescence spectra of the Rh/ITO sample could not be measured using our optical system because its intensity was very low. It is thought that holes were effectively injected from an anode to an organic layer, promoting internal quantum efficiency.

Figure 2 shows the $\mathrm{x}$-ray reflectivity curves of $\mathrm{Rh} / \mathrm{ITO}$ and $\mathrm{O}_{2}-\mathrm{Rh} / \mathrm{ITO}$ samples. The thickness of the Rh layer estimated from the x-ray reflectivity data was $2 \mathrm{~nm}$. The film thickness is given by $2 \pi / \Delta q$, where $\Delta q$ is the period of the intensity oscillations in the reflectivity curves. ${ }^{10}$ The amplitude of the intensity oscillation in the $\mathrm{O}_{2}-\mathrm{Rh} / \mathrm{ITO}$ sample is higher than that in the Rh/ITO sample. The intensity oscillation reflects the interference pattern of $\mathrm{x}$ rays reflected from the surface and the interface. Therefore, it is considered that the Rh surface became smoother after $\mathrm{O}_{2}$ plasma treatment.

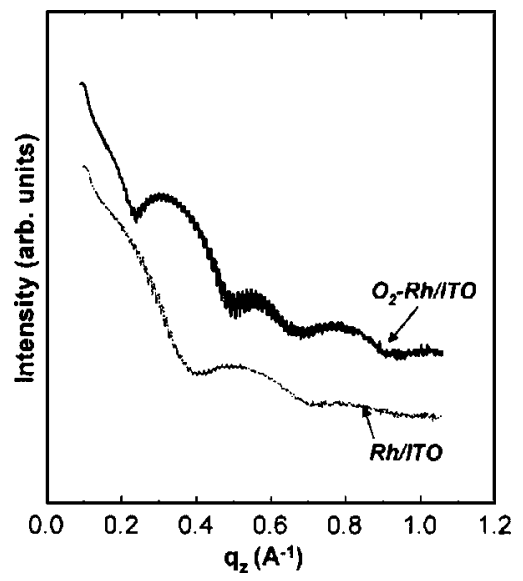

FIG. 2. X-ray reflectivity data of $\mathrm{O}_{2}-\mathrm{Rh} / \mathrm{ITO}$ and $\mathrm{Rh} / \mathrm{ITO}$.

Figure 3(a) shows O $1 s$ SRPES spectra for three kinds of samples. In order to separate the chemical bonding states including those in the spectra, the spectral line shape was simulated using a suitable combination of Gaussian and Lorentzian functions. The $\mathrm{O} 1 \mathrm{~s}$ peak was separated into two components. The P1 peak centered at $532.2 \mathrm{eV}$ in ITO was due to residual surface contaminants. ${ }^{11}$ The $\mathrm{P} 2$ peak centered at $530 \mathrm{eV}$ was assigned to $\mathrm{O}^{2-}$ ions in the tetrahedral interstices of face-centered cubic $\operatorname{In}^{3+}$ ion arrays. ${ }^{12}$ In the case of $\mathrm{Rh} / \mathrm{ITO}$, the peak intensity of $\mathrm{O} 1 s$ decreased. The photoemission flux $I_{d}$ penetrating a film decreased exponentially with the increase of thickness $d$, expressed as $I_{d}=$ $I_{0} \exp (-d / \lambda)$, where $I_{0}$ is the flux emitted by the clean backing material and $\lambda$ is the inelastic mean-free path of an electron traveling within a solid. ${ }^{13}$ The reduction of $\mathrm{O} 1 \mathrm{~s}$ peak intensity in the $\mathrm{Rh} / \mathrm{ITO}$ is due to the exponential decrease of photoemission flux. In $\mathrm{O}_{2}-\mathrm{Rh} / \mathrm{ITO}$, a new $\mathrm{P} 3$ peak centered at $529.5 \mathrm{eV}$ appeared, indicating the $\mathrm{O}-\mathrm{Rh}$ bond. Therefore, it is thought that $\mathrm{O}_{2}$ plasma treatment transformed the Rh layer into a transparent $\mathrm{RhO}_{x}$ layer. The $\mathrm{Rh} 3 d$ core level spectra of $\mathrm{Rh} / \mathrm{ITO}$ and $\mathrm{O}_{2}-\mathrm{Rh} / \mathrm{ITO}$ are shown in Fig. 3(b). The $\mathrm{Rh} 3 d_{5 / 2}$ and $\mathrm{Rh} 3 d_{3 / 2}$ peaks shifted about $1.5 \mathrm{eV}$ toward a higher binding energy after $\mathrm{O}_{2}$ plasma treatment. It is reported that the binding energy of the Rh-O bond is 1.4 $\sim 1.6 \mathrm{eV}$ higher than that of the Rh-Rh bond. ${ }^{14}$ Thus, the shift of $\mathrm{Rh} 3 d_{5 / 2}$ and $\mathrm{Rh} 3 d_{3 / 2}$ peaks is due to the formation of $\mathrm{RhO}_{x}$ layers.

The relative change of the work function was measured using secondary electron emission spectra, as shown in Fig.

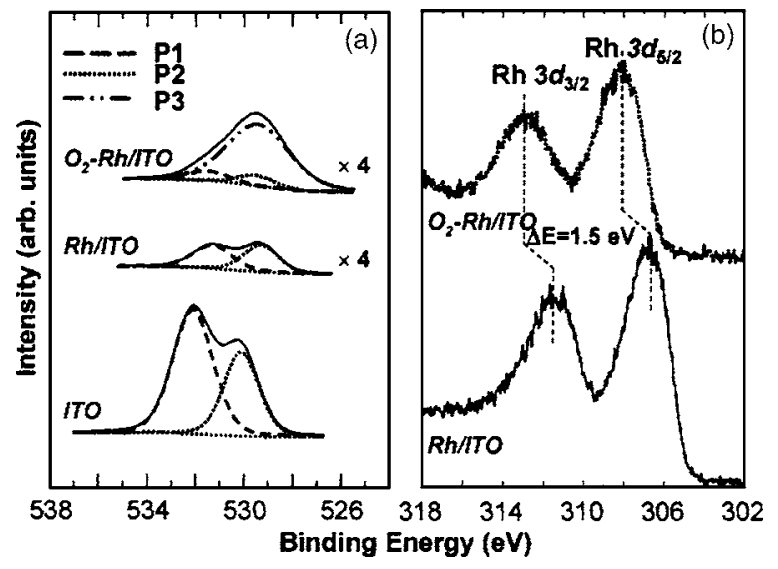

FIG. 3. (a) $\mathrm{O} 1 s$ and (b) Rh $3 d$ core level spectra for three kinds of anodes. 


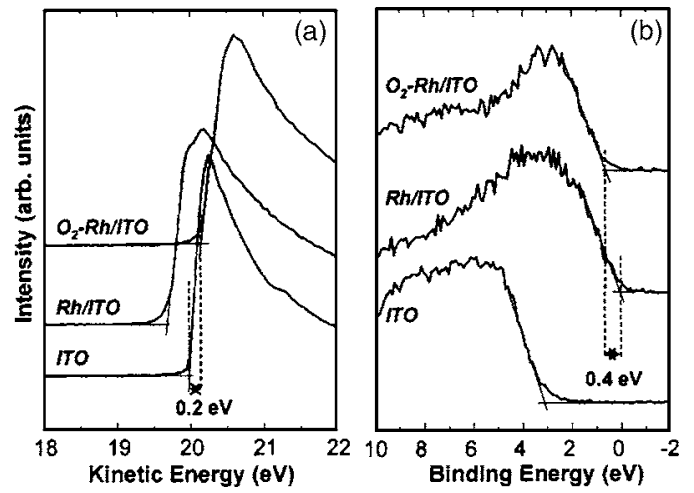

FIG. 4. (a) Secondary electron-emission spectra and (b) relative change of valence band maximum for the samples.

4(a). The onset of secondary electrons was determined by extrapolating two solid lines from the background and straight onset in the spectra. ${ }^{15}$ The onset of a secondary electron for $\mathrm{O}_{2}-\mathrm{Rh} / \mathrm{ITO}$ shifted to the higher kinetic energy by $0.2 \mathrm{eV}$ with respect to the onset for ITO. This result means that the work function of $\mathrm{RhO}_{x}$-coated ITO is higher by 0.2 $\mathrm{eV}$ than that of $\mathrm{O}_{2}$ plasma-treated ITO. Figure 4(b) shows the relative change in the valence band maximum (VBM) with the formation of a $\mathrm{RhO}_{x}$ layer. The VBM was calibrated with a clean Au surface. The VBM at the ITO surface is located $3.0 \mathrm{eV}$ apart from the Fermi level. After the Rh layer deposition on the ITO surface, the VBM of Rh/ITO coincided with the Fermi level, showing the metallic valance band. However, the VBM of $\mathrm{O}_{2}-\mathrm{Rh} / \mathrm{ITO}$ is located at $0.4 \mathrm{eV}$, apart from the Fermi level. This means that the bandgap was produced due to the formation of $\mathrm{RhO}_{x}$. This is in good agreement with the result of Fig. 3.

Based on these experimental observations, the reduction in operation voltage could be explained as later. The electron concentration of ITO is about $10^{20}-10^{21} \mathrm{~cm}^{-3}$. ${ }^{16}$ Thus, the Fermi level of ITO is close to the conduction band maximum due to its high electron concentration. After $\mathrm{O}_{2}$ plasma treatment, surface band bending increased, as shown in Fig. 5(a). This could be due to the formation of the Sn-deficient and O-rich surface by the treatment. ${ }^{6}$ When the Rh layer changed

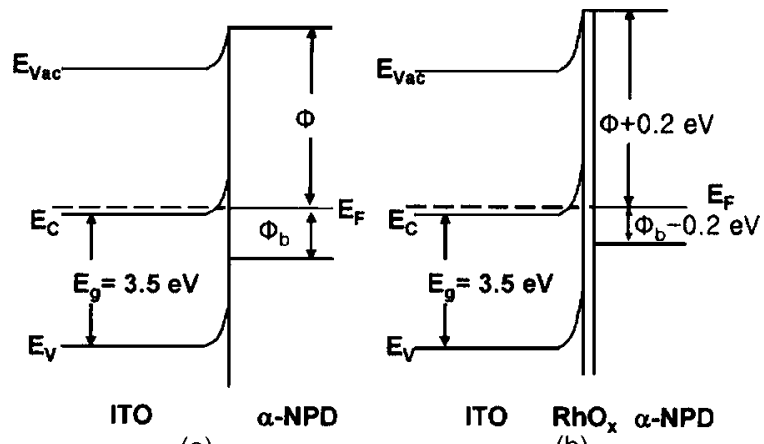

(a)

(b)

FIG. 5. Schematic band diagram: (a) $\mathrm{O}_{2}$ plasma-treated ITO and (b) $\mathrm{O}_{2}$ plasma-treated Rh layer on ITO. to a transparent $\mathrm{RhO}_{x}$ layer by $\mathrm{O}_{2}$ plasma treatment, the work function increased [Fig. 4(a)], leading to the increase in band bending, as shown in Fig. 5(b). The injection barrier for holes from anodes to organic materials corresponds to the energy difference between the work function of anodes and ionization energy of organic materials. Thus, the hole injection barrier of ITO, $\Phi_{b}$, decreased to $\Phi_{b}-0.2 \mathrm{eV}$ with $\mathrm{O}_{2}$ plasma treatment. Therefore, the interfacial layer of $\mathrm{RhO}_{x}$ plays a role in lowering the potential barrier between ITO and organic materials, reducing the turn-on voltage of OLEDs.

In conclusion, we have reported the advantage of interfacial $\mathrm{RhO}_{x}$ layers between anodes of ITOs and hole transport layers of $\alpha$-NPD on the electrical properties of OLEDs. The turn-on voltage of OLEDs decreased from 7 to $5 \mathrm{~V}$ and the operation voltage at $700 \mathrm{~cd} / \mathrm{m}^{2}$ decreased from 13 to 10 $\mathrm{V}$ as the $\mathrm{RhO}_{x}$ layer exists between ITO and $\alpha$-NPD. The $\mathrm{RhO}_{x}$ layer had a higher work function than ITO by $0.2 \mathrm{eV}$. Thus, the $\mathrm{RhO}_{x}$ layer lowered the potential barrier for hole injection from ITO to $\alpha$-NPD, reducing the turn-on voltage of OLEDs and increasing quantum efficiency.

This research was supported in part by the Program for the Training of Graduate Students in Regional Innovation that was conducted by the Ministry of Commerce, Industry, and Energy of the Korean Government, in part by Korea Science and Engineering Foundation through the QuantumFunctional Semiconductor Research Center at Dongguk University in 2005, and in part by the project for the National Research Laboratory supported by the Korea Institute of Science and Technology Evaluation and Planning (KISTEP).

${ }^{1}$ G. Liu, J. B. Kerr, and S. Johnson, Synth. Met. 144, 1 (2004).

${ }^{2}$ L. S. Hung, C. W. Tang, and M. G. Mason, Appl. Phys. Lett. 70, 13 (1997).

${ }^{3}$ M. Y. Chan, S. L. Lai, M. K. Fung, S. W. Tong, C. S. Lee, and S. T. Lee, Appl. Phys. Lett. 82, 1784 (2003).

${ }^{4}$ S.-F. Chen and C.-W. Wang, Appl. Phys. Lett. 85, 765 (2004).

${ }^{5}$ S. W. Tong, C. S. Lee, Y. Lifshitz, D. Q. Gao, and S. T. Lee, Appl. Phys. Lett. 84, 4032 (2004).

${ }^{6}$ K. H. Lee, H. W. Jang, K.-B. Kim, Y.-H. Tak, and J.-L. Lee, J. Appl. Phys. 95, 586 (2004).

${ }^{7}$ I.-M. Chan and F. C. Hong, Thin Solid Films 450, 304 (2004).

${ }^{8}$ C.-M. Hsu and W.-T. Wu, Appl. Phys. Lett. 85, 840 (2004).

${ }^{9}$ D. R. Lide, CRC Handbook of Chemistry and Physics, 83rd ed. (CRC Press, Boca Raton, FL, 2002).

${ }^{10}$ D. Y. Noh, Y. Hwu, H. K. Kim, and M. Hong, Phys. Rev. B 51, 4441 (1995).

${ }^{11}$ L. Chkoda, C. Heske, M. Sokolowski, E. Umbach, F. Steuber, J. Staudigel, M. Stobel, and J. Simmerer, Synth. Met. 111, 315 (2000).

${ }^{12}$ W. R. Salaneck, N. Johansson, K. Z. Xing, F. Cacialli, R. H. Friend, G. Beamson, and D. T. Clark, Synth. Met. 92, 207 (1998).

${ }^{13}$ D. J. O'Connor, B. A. Sexton, and R. St. C. Smart, Surface Analysis Methods in Materials Science (Springer-Verlag, Berlin, 1992).

${ }^{14}$ J. F. Moulder, W. F. Strickle, P. E. Sobol, and K. D. Bomben, Handbook of $X$-Ray Photoelectron Spectroscopy (Perkin-Elmer, Eden Prairie, MN, 1992).

${ }^{15}$ J. M. Baik and J.-L. Lee, Met. Mater. Int. 10, 555 (2004).

${ }^{16}$ A. K. Kulkarni and S. A. Knickerbocker, J. Vac. Sci. Technol. A 14, 1709 (1996). 